\title{
Lobelia siphilitica Root Oil
}

National Cancer Institute

\section{Source}

National Cancer Institute. Lobelia siphilitica Root Oil. NCI Thesaurus. Code C74284.

The oil extracted from the roots of Lobelia siphilitica. 